\title{
BIFURCATION OF STATIONARY AND HETEROCLINIC ORBITS FOR PARAMETRIZED GRADIENT-LIKE FLOWS
}

\author{
THOMAS BARTSCH \\ Mathematisches Institut, Universität Heidelberg, \\ Im Neuenheimer Feld 288, 69120 Heidelberg, Germany \\ E-mail: bartsch@harmless.mathi.uni-heidelberg.de
}

1. Introduction. In this paper we study two bifurcation problems which are in a sense complementary or dual to each other. Our goal is to show how methods from Conley index theory and minimax theory can be combined in order to provide a unified approach to both types of problems. We also hope to provide an introduction to recent ideas in bifurcation theory. Moreover we obtain classical bifurcation results due to Böhme, Marino, Fadell, Rabinowitz and others as corollaries. For the sake of exposition we shall not present the most general versions of our results. Also some technical details of the proofs have to be skipped due to lack of space.

We first consider the following situation. Let $G: \mathbf{R}^{n} \rightarrow \mathbf{R}$ be of class $\mathcal{C}^{2}$ and let $\varphi$ be a (local) flow on $\mathbf{R}^{n}$ which leaves the level sets $G^{-1}(c)$ invariant. Suppose moreover that $\varphi$ is gradient-like, that is, there is a Lyapunov function $F: \mathbf{R}^{n} \rightarrow \mathbf{R}$ such that $F(\varphi(x, t))$ is strictly decreasing in $t$ for every nonstationary point $x \in \mathbf{R}^{n}$. This has the effect that the only bounded orbits of $\varphi$ are stationary orbits and heteroclinic orbits. Here an orbit is called heteroclinic if both its $\alpha$ - and $\omega$-limit sets are nonempty and disjoint. These limit sets necessarily consist only of stationary orbits. Next we assume that the origin is a non-degenerate critical point of $G$ with critical value $G(0)=0$ and Morse index $\mu$. If $\mu \neq n / 2$, for instance if $n$ is odd, then the level sets $G^{-1}(c)$ change their topological type (in a neighborhood of the origin) as $c$ passes 0 . More precisely, $G^{-1}(c) \cap U(0)$ is diffeomorphic to $S^{\mu-1} \times D^{n-\mu}$ for $c<0$ and to $D^{\mu} \times S^{n-\mu-1}$ for $c>0$, provided $U(0)$ is properly chosen. Clearly, the level set $G^{-1}(0)$ has a singularity at the origin. This implies that 0 is a stationary orbit of $\varphi$. Since we are only interested in the local situation near the origin we do not care about other possible singularities on $G^{-1}(c)$ away from a neighborhood of 0 . We shall see that, without any additional assumptions on

1991 Mathematics Subject Classification: Primary 58E07; Secondary 58E40, 47H17

The paper is in final form and no version of it will be published elsewhere. 
$\varphi$, this local change of topology forces the existence of other stationary orbits of $\varphi$ near the origin. Moreover we shall be interested in the direction of the bifurcating stationary solutions (whether they lie on $G^{-1}(c)$ with $c>0, c=0$ or $c<0$ ). The strongest results will be obtained when $G$ is even and $\varphi$ is odd with respect to the $x$-variable. We can also prove the existence of bifurcating heteroclinic orbits on certain level sets. Extensions to more general symmetries will be mentioned.

The second situation which we want to study is a more classical bifurcation setting. Here $\varphi_{\lambda}$ is a family of flows on $\mathbf{R}^{n}$ continuously parametrized by a real parameter $\lambda \in \mathbf{R}$. Again we assume that $\varphi_{\lambda}$ is gradient-like for each $\lambda$. Suppose that the origin is a stationary orbit of $\varphi_{\lambda}$ for every $\lambda$. The points $(\lambda, 0)$ are called trivial orbits. We are interested in the bifurcation of nontrivial bounded orbits from the set of trivial orbits. Fixing some possible bifurcation parameter $\lambda_{0}$ we assume that the origin is a non-degenerate stationary orbit of $\varphi_{\lambda}$ for $\lambda$ close to $\lambda_{0}$ but different from $\lambda_{0}$. Then it is well known that bifurcation must occur if the origin changes stability as $\lambda$ passes $\lambda_{0}$, that is, if the dimension of the unstable manifold of the origin with respect to $\varphi_{\lambda}$ changes. We shall investigate more closely the structure of the bifurcating set of stationary and heteroclinic orbits, in particular in the case when $\varphi_{\lambda}$ is odd in the space variable. Again the case of more general symmetries will be sketched.

Both situations can be put into a more abstract setting which shows their relation. Let $(\Sigma, \pi)$ be a locally compact topological space over the parameter space $\Lambda$, i.e. $\pi: \Sigma \rightarrow \Lambda$ is a continuous surjective map. For simplicity we only consider the case where $\Lambda \subset \mathbf{R}$ is an interval. We are given a gradient-like flow over $\Lambda$, that is, a flow on $\Sigma$ which respects the "fibres" $\Sigma_{\lambda}:=\pi^{-1}(\lambda)$, for any $\lambda \in \Lambda$. In the first situation $\Sigma=\mathbf{R}^{n}$ and $\pi=G$. The existence of bounded solutions near a stationary solution $x_{0} \in \Sigma_{0}$ comes from a local change of the topology of the fibres near $x_{0}$ due to the fact that $x_{0}$ is a critical point of $\pi$. In other words, the behaviour of $\pi$ forces bifurcation without any additional assumptions on $\varphi$. In the second situation $\Sigma=\mathbf{R} \times \mathbf{R}^{n}$ and $\pi$ is the projection onto the first factor. Obviously this $\pi$ has no critical points at all. If $x_{0} \in \Sigma_{0}$ is a stationary solution of $\varphi_{\lambda_{0}}$ then in general we can neither expect that this solution can be continued for other parameter values nor that there exist nontrivial orbits if a trivial branch exists. Thus we have to make assumptions on the behavior of the flow $\varphi$ in order to have bounded solutions bifurcating from a trivial branch.

One motivation for studying these bifurcation settings are nonlinear eigenvalue problems. Let $F, G: X \rightarrow \mathbf{R}$ be of class $\mathcal{C}^{2}$ defined on the Banach space $X$. For simplicity $X=\mathbf{R}^{n}$. We are interested in solutions of the equation $(*) F^{\prime}(x)=\lambda G^{\prime}(x)$. Suppose the origin $0 \in X$ is a critical point of $F$ and $G$. In other words, $(\lambda, 0)$ is a solution of $(*)$ for every $\lambda \in \mathbf{R}$. Then we may try to parametrize bifurcating nontrivial solutions using the level sets of $G$ as parameter. In this approach $\lambda$ appears as Lagrange multiplier and cannot be prescribed. We have to study the negative gradient flow of $F \mid G^{-1}(c)$ for $c$ near $G(0)$. Thus we arrive at the first situation described above. Or we may try to use $\lambda$ as parameter for the bifurcating solutions which leads us to the second situation from above. Here we cannot control the level sets on which the nontrivial solutions lie. The parameters $c$ and $\lambda$ play a dual role in this problem. 
The paper is organized as follows. In $\S 2$ we state and discuss our main results concerning the first setting where a change of topology forces bifurcation. In $\S 3$ we treat the complementary situation where the topology of the space does not change but a change of stability yields bifurcation. Then in $\S 4$ we apply these results to nonlinear eigenvalue problems. This section mainly serves as a motivation for studying our bifurcation problems. We have to refer to the literature for parts of the proofs. In $\S 5$ we collect some basic definitions from Conley index theory and topology (genus, cohomological index) which we need in the proofs. Finally $\S \S 6,7$ contain sketches of the proofs of our bifurcation theorems.

2. Bifurcation forced by a change of topology. Let $G: U \rightarrow \mathbf{R}$ be of class $\mathcal{C}^{2}$, defined on an open neighborhood $U$ of $0 \in \mathbf{R}^{n}$ such that the only critical point of $G$ is the origin which is non-degenerate with Morse index $\mu$. We may assume that $G(0)=0$. Let $\varphi$ be a local flow on $U$ which respects the level sets of $G$. Thus

$$
\varphi: \mathcal{O} \rightarrow \mathbf{R}^{n},(x, t) \mapsto \varphi(x, t)=\varphi^{t}(x)
$$

is a continuous map defined on an open subset $\mathcal{O} \subset \mathcal{U} \times \mathbf{R}$ such that $\{t \in \mathbf{R}:(x, t) \in \mathcal{O}\}$ is an open interval containing 0 for each $x \in U$. It satisfies $\varphi^{0}(x)=x$ and $\varphi^{s+t}(x)=$ $\varphi^{s} \circ \varphi^{t}(x)$ for $x \in U$ and $s, t \in \mathbf{R}$, if these expressions are defined. Moreover, $G\left(\varphi^{t}(x)\right)$ is independent of $t$, for any $x \in U$. In other words, $G$ is a first integral for $\varphi$.

The Morse lemma tells us that after a change of coordinates near the origin, we may assume that $U$ is a small ball around the origin and $G(y, z)=\|z\|^{2}-\|y\|^{2}$ where $(y, z) \in U \subset \mathbf{R}^{\mu} \times \mathbf{R}^{n-\mu}$. Thus $G^{-1}(c)$ is diffeomorphic to $S^{\mu-1} \times D^{n-\mu}$ if $c<0$ and to $D^{\mu} \times S^{n-\mu-1}$ if $c>0$ where $D^{k}$ denotes the open unit ball of dimension $k$ and $|c|$ is small. The level set $G^{-1}(0)$ is a manifold with a singularity at 0 . It is diffeomorphic to $S^{\mu-1} \times D^{n-\mu} / S^{\mu-1} \times\{0\}$ or to $D^{\mu} \times S^{n-\mu-1} /\{0\} \times S^{n-\mu-1}$. Since the maps $\varphi^{t}$ are homeomorphisms it follows that the origin is a stationary point of the flow, that is, $\varphi^{t}(0)=0$ for every $t \in \mathbf{R}$.

Finally we assume that $\varphi$ is gradient-like, that is, there is a continuous function $F: U \rightarrow \mathbf{R}$ such that $F\left(\varphi^{t}(x)\right)$ is strictly decreasing in $t$ for every $x \in U$ except when $x$ is a stationary point. Now we can state our first result.

THEOREM 2.1. If $2 \mu \neq n$ then at least one of the following holds.

(i) There exists a sequence $x_{k} \in G^{-1}(0), k \in \mathbf{N}$, of stationary points of $\varphi$ which converges towards the origin as $k \rightarrow \infty$.

(ii) For each $c>0$ close to 0 there exists a stationary point $x_{c} \in G^{-1}(c)$ of $\varphi$ which converges towards the origin as $c \rightarrow 0$.

(iii) The same statement as in (ii) holds for $c<0$ close to 0.

Clearly the assumption $2 \mu \neq n$ is always satisfied for odd $n$. It guarantees that the level sets $G^{-1}(c)$ change topology as $c$ passes 0 . The following examples show that this assumption is necessary and that each possibility listed in the theorem may occur. 
ExAMPLE 2.2. a) We consider the case $2 \mu=n$ and $G(y, z)=\|z\|^{2}-\|y\|^{2}$ with $(y, z) \in \mathbf{R}^{\mu} \times \mathbf{R}^{\mu}=\mathbf{R}^{n}$. Setting

$$
F(y, z):=\sum_{i=1}^{\mu}\left(y_{i}^{2}+z_{i}^{2}\right) \cdot \sum_{i=1}^{\mu} y_{i} z_{i}
$$

we define a vector field $\nabla_{G} F$ on $\mathbf{R}^{n}$ as follows: $\nabla_{G} F(0):=0$ and, for $x \neq 0, \nabla_{G} F(x)$ is the projection of $\nabla F(x)$ onto $T_{x} G^{-1}(c)$ where $c=G(x)$. Thus

$$
\nabla_{G} F(x):=\nabla F(x)-\frac{\langle\nabla F(x), \nabla G(x)\rangle}{\|\nabla G(x)\|^{2}} \cdot \nabla G(x)
$$

for $x \in \mathbf{R}^{n}-0$. This vector field can be integrated since it is locally Lipschitz in $\mathbf{R}^{n}-0$. The associated flow $\varphi$ which satisfies $\frac{d}{d t} \varphi(x, t)=\nabla_{G} F(\varphi(x, t))$ is also continuous at the points $(0, t)$, any $t \in \mathbf{R}$. It preserves the level sets of $G$ since it is the negative gradient flow of $F \mid G^{-1}(c)$ on each level set $G^{-1}(c)-0$. We claim that there are no other stationary points except the origin. In order to see this we first observe that a stationary point $x=(y, z) \in \mathbf{R}^{n}-0$ satisfies $\nabla_{G} F(x)=0$. We take the scalar product of this equation with $(z, y)$, use the fact that $\langle\nabla G(x),(z, y)\rangle=0$ and obtain:

$$
0=\left\langle\nabla_{G} F(x),(z, y)\right\rangle=\langle\nabla F(x),(z, y)\rangle=4\left(\sum_{i=1}^{\mu} y_{i} z_{i}\right)^{2}+\|x\|^{4}
$$

This implies $x=0$, hence there are no nontrivial stationary points at all. This also implies that the origin is the only bounded orbit.

b) Let $n=3$ and $G(x)=x_{1}^{2}+x_{2}^{2}-x_{3}^{2}$, thus $\mu=1$. We define

$$
F(x):= \begin{cases}x_{1} G(x) & \text { if } G(x)<0 \\ 0 & \text { if } G(x)=0 \\ x_{3} G(x) & \text { if } G(x)>0\end{cases}
$$

and let $\varphi$ be the flow obtained by integrating the vector field $\nabla_{G} F$. Then again stationary points $x \in \mathbf{R}^{n}-0$ of $\varphi$ satisfy $\nabla_{G} F(x)=0$. Clearly $\nabla_{G} F(x)=0$ for every $x \in G^{-1}(0)$. On $G^{-1}(c), c<0$, we have $F(x)=c x_{1}$, and it is easy to check that this function has no critical points on $G^{-1}(c)$. The same is true for $c>0$. Thus in this example only alternative (i) from Theorem 2.1 occurs. One can turn this example into a smooth one upon replacing $F(x)$ by $F(x) \cdot \exp \left(-1 / G(x)^{2}\right)$

c) We take $G$ as in b) and let $\varphi$ be the flow obtained by integrating $\nabla_{G} F$ where

$$
F(x)=x_{1}^{4}+x_{2}^{3} x_{3}+x_{2} x_{3}^{3}
$$

Taking the scalar product of the equation $\nabla_{G} F(x)=0$ with $\left(0, x_{3}, x_{2}\right)$ we obtain $x_{2}=$ $x_{3}=0$. The only stationary orbits of $\varphi$ are the points $\left(x_{1}, 0,0\right), x_{1} \in \mathbf{R}$, which lie on $G^{-1}(c)$ for $c=x_{1}^{2}>0$, so only alternative (ii) from Theorem 2.1 applies.

d) In our last example $G$ is as in b) and $\varphi$ is the flow associated to $\nabla_{G} F$ with

$$
F(x)=x_{3}^{3}+x_{1}^{2} x_{3}+x_{2}^{2} x_{3}
$$

Elementary calculations as above show that the only stationary orbits of $\varphi$ are the points $\left(0,0, x_{3}\right), x_{3} \in \mathbf{R}$, which lie on $G^{-1}(c)$ for $c=-x_{3}^{2}<0$, thus only alternative 2.1(iii) holds true. 
An interesting question is whether one can obtain a multiplicity result. Suppose 2.1(i) is false. If both (ii) and (iii) apply then we have two bifurcating "branches". However, if only (ii) holds true, then one may ask whether there always exist two stationary solutions on $G^{-1}(c)$ for $c>0$. Observe that in the example 2.2c) this is the case. One can show that this is always true in the cases $\mu=0,1, n-1, n, n \geq 3$. Thus the lowest dimensional example with precisely one stationary orbit on each positive level set and no solution on nonpositive level sets can exist for $n=5$ and $\mu=2$ or $\mu=3$. These stationary orbits have to be degenerate. For such an example one has to leave the realm of polynomial (or analytic) maps.

As an aside, in the example 2.2c) there are no connecting orbits between the bifurcating stationary orbits on $G^{-1}(c)$ because the stationary orbits $( \pm \sqrt{c}, 0,0)$ satisfy $F( \pm \sqrt{c}, 0,0)=c^{2}$. Also in $\left.2.2 \mathrm{~d}\right)$ there are no connecting orbits between the stationary orbits $(0,0, \pm \sqrt{-c})$ on $G^{-1}(c)$ because these orbits lie in different connected components of $G^{-1}(c)$. Thus in 2.1b), c) there need not exist other solutions whose closure in $U$ is compact. On the other hand, if only 2.1a) applies then the set of relatively compact (in $U$ ) orbits of $\varphi$ contained in $G^{-1}(0)$ connects the origin to the boundary of $U$. More precisely, the proof of Theorem 2.1 in $\S 6$ shows that, if $2.1 \mathrm{~b}$ ), c) do not hold then there does not exist a compact neighborhood $N \subset G^{-1}(0)$ of the origin which is isolating in the sense of Conley index theory.

Now we consider the same situation with an additional symmetry condition. For simplicity we stick to the case where $G$ is an even function and $\varphi$ is odd: $G(-x)=G(x)$, so the level surfaces of $G$ are symmetric with respect to the reflection $x \mapsto-x$ and $\varphi^{t}(-x)=-\varphi^{t}(x)$. The case of more general symmetries will be discussed below. Classical results from critical point theory suggest that symmetry forces the existence of multiple solutions. In order to formulate our next result we recall the notion of genus for symmetric subspaces of $\mathbf{R}^{n}$ due to Krasnoselski $[\mathrm{K}]$ and Yang $[\mathrm{Y}]$.

Definition 2.3. Let $A \subset \mathbf{R}^{n}$ be symmetric, i.e. invariant with respect to the reflection $x \mapsto-x$. The genus of $A$, genus $(A)$, is the least natural number $k$ such that there exists an odd continuous map $\alpha: A \rightarrow S^{k-1}$. If $0 \in A$ then no such map can exist and we set $\operatorname{genus}(A)=\infty$. For $A=\emptyset$ we have genus $(\emptyset)=0$.

Clearly we have $1 \leq \operatorname{genus}(A) \leq n$ for every nonempty subset $A$ of $\mathbf{R}^{n}-0$. Moreover, $\operatorname{genus}\left(S^{k-1}\right)=k$ where $S^{k-1}$ is the unit sphere in $\mathbf{R}^{k} \subset \mathbf{R}^{n}$. This is a consequence of Borsuk's theorem on the degree of odd maps between spheres.

THEOREM 2.4. At least one of the following is true:

(i) There exists a sequence $\pm x_{k} \in G^{-1}(0), k \in \mathbf{N}$, of stationary points of $\varphi$ which converges towards the origin as $k \rightarrow \infty$.

(ii) For each $c$ close to 0 with $c \cdot(n-2 \mu)>0$ there exists a compact subset $S \subset G^{-1}(c)$ which is invariant under the flow, symmetric and satisfies $\operatorname{genus}(S) \geq|n-2 \mu|$. S contains at least $|n-2 \mu|$ antipodal pairs $\pm x$ of stationary orbits. Moreover, if there are only finitely many stationary orbits on $S$ then there exist $|n-2 \mu|$ such stationary pairs $\pm x_{i}$ and connecting orbits from $x_{i+1}$ to $x_{i} . S$ converges towards the origin as $c \rightarrow 0$. 
If $n=2 \mu$ then part b) holds trivially with $S=\emptyset$. The example 2.2a) shows that $n \neq 2 \mu$ is necessary for bifurcation also in the symmetric case. At first sight it is somewhat surprising that in the symmetric case the sign of $n-2 \mu$ determines the direction of the bifurcating set. Looking back at the examples in 2.2 we see that the third example is symmetric but the last one is not. The third example corresponds to 2.4(ii) whereas in the last example the bifurcating solutions lie on the "wrong" energy levels: $n-2 \mu>0$ but there are only solutions on negative energy levels. Theorem 2.4 shows that it is not possible to find an even map $F: \mathbf{R}^{3} \rightarrow \mathbf{R}$ which realizes alternative 2.1(iii) with $G$ as in $2.2 \mathrm{~d}$ ). It is also possible to construct an even example which realizes $2.4(\mathrm{i})$. This is somewhat more complicated to describe than the one in $2.2 \mathrm{~b}$ ) and is therefore left to the interested reader.

It is possible to analyze $S$ and the flow on $S$ further. For instance, one can prove that $\operatorname{dim} S \geq|n-2 \mu|$ and one can obtain results on the dimensions of the unstable manifolds of the stationary orbits and on the dimensions of the sets of connecting orbits between $x_{i+1}$ and $x_{i}$.

Remark 2.5. One can also deal with other symmetries than the reflection at the origin. Suppose a compact Lie group $\Gamma$ acts orthogonally on $\mathbf{R}^{n}$ and $G$ is invariant, $\varphi$ is equivariant. This means $G(\gamma x)=G(x)$ and $\varphi^{t}(\gamma x)=\gamma \varphi^{t}(x)$ for every $\gamma \in \Gamma$, $(x, t) \in \mathcal{O} \subset U \times \mathbf{R}$. Clearly a stationary orbit $x$ of $\varphi$ gives rise to a whole $\Gamma$-orbit $\{\gamma x: \gamma \in \Gamma\}$ of stationary points. This $\Gamma$-orbit lies on one level set of $G$. In that case we can refine the assumptions of Theorem 2.1 and prove an analogue of Theorem 2.4. Let $\mathbf{R}^{n} \cong V^{+} \oplus V^{-}$be the decomposition of $\mathbf{R}^{n}$ according to the positive respectively the negative part of the spectrum of $G^{\prime \prime}(0)$. The invariance of $G$ implies that $V^{+}$and $V^{-}$are invariant under the action of $\Gamma$, hence, they are representation spaces of $\Gamma$. Two representations $V_{1}, V_{2}$ of $\Gamma$ are said to be $\Gamma$-homotopy equivalent if their one-point compactifications $V_{1} \cup\{\infty\}$ and $V_{2} \cup\{\infty\}$ are $\Gamma$-homotopy equivalent with the base points $\infty$ being fixed. $V_{1}$ and $V_{2}$ are said to be stable $\Gamma$-homotopy equivalent if there exists a representation $W$ of $\Gamma$ such that $V_{1} \oplus W$ and $V_{2} \oplus W$ are $\Gamma$-homotopy equivalent. Clearly, isomorphic representations are $\Gamma$-homotopy equivalent. The converse is false in general; cf. [CaS]. One can prove that the conclusion of Theorem 2.1 remains valid in the $\Gamma$ equivariant case provided $V^{+}$and $V^{-}$are not stable $\Gamma$-homotopy equivalent. This is much weaker than the assumption $\operatorname{dim} V^{+} \neq \operatorname{dim} V^{-}$. For instance, consider the case $\Gamma=\mathbf{Z} / p$ where $p$ is a prime. For $j=0, \ldots, p-1$ let $V_{j} \cong \mathbf{C}$ be the irreducible representation of $\Gamma$ with the character $\gamma \mapsto \gamma^{j}$. Here we think of $\mathbf{Z} / p$ as a subset of the group of complex numbers of modulus 1 . Then $V_{j}$ and $V_{k}$ are not stable homotopy equivalent for $j \neq k$. It is an interesting problem whether it suffices that $V^{+}$and $V^{-}$are not $\Gamma$-homotopy equivalent or only non-isomorphic.

It is also possible to define a version of genus for $\Gamma$-invariant subsets of $\mathbf{R}^{n}$. The important special case $\Gamma=S^{1}$ is due to Benci [Be], the general case can be found in [Ba1] or [Ba2], §2.3. Then Theorem 2.4 generalizes to the $\Gamma$-equivariant case with $n-2 \mu$ in (ii) replaced by genus $\left(S V^{+}\right)-\operatorname{genus}\left(S V^{-}\right)$. Of course we also replace the stationary pairs $\pm x$ by $\Gamma$-orbits $\{\gamma x: \gamma \in \Gamma\}$ of stationary points. In the case $\Gamma=\mathbf{Z} / p$ acting without fixed points on $\mathbf{R}^{n}-0$ one has genus $\left(S V^{ \pm}\right)=\operatorname{dim} V^{ \pm}$so Theorem 2.4 
generalizes verbatim. In the case $\Gamma=S^{1}$ acting without fixed points on $\mathbf{R}^{n}-0$ one has $\operatorname{genus}\left(S V^{ \pm}\right)=\left(\operatorname{dim} V^{ \pm}\right) / 2$ so $n-2 \mu$ in 2.4 has to be replaced by $(n-2 \mu) / 2$. The proof of 2.4 uses only the properties of the genus as an index theory (these properties will be recalled in $\S 5$ below). Thus any index theory instead of the genus will work. In particular, one can use the length, which is an index theory defined with the help of cohomology (see $\S 5$ below and [Ba2], 44.3 ).

3. Bifurcation forced by a change of stability. Let $\varphi_{\lambda}$ be a continuous family of gradient-like flows on $\mathbf{R}^{n}$, parametrized by $\lambda \in \mathbf{R}$. We assume that the origin is a stationary orbit of $\varphi_{\lambda}$ for every $\lambda$. We fix some parameter value $\lambda_{0}$ so that 0 is a degenerate stationary point of $\varphi_{\lambda_{0}}$ and a hyperbolic stationary point of $\varphi_{\lambda}$ for $\lambda \neq \lambda_{0}$ close to $\lambda_{0}$. Let $m_{l}^{u}$ respectively $m_{r}^{u}$ denote the dimension of the unstable manifold of 0 with respect to $\varphi_{\lambda}$ for $\lambda<\lambda_{0}$ respectively $\lambda>\lambda_{0}$ close to $\lambda_{0}$. These numbers are well defined, that is, independent of the particular choice of $\lambda$. The first result which corresponds to Theorem 2.1 is well known.

THEOREM 3.1. If $m_{l}^{u} \neq m_{r}^{u}$ then at least one of the following holds.

(i) There exists a sequence $\left(x_{k}: k \in \mathbf{N}\right)$ of nontrivial stationary points of $\varphi_{\lambda_{0}}$ which converges towards the origin as $k \rightarrow \infty$.

(ii) For each $\lambda>\lambda_{0}$ close to $\lambda_{0}$ there exists a stationary point $x_{\lambda} \neq 0$ of $\varphi_{\lambda}$ which converges towards the origin as $\lambda \rightarrow \lambda_{0}$.

(iii) The same statement as in (ii) holds for $\lambda<\lambda_{0}$ close to $\lambda_{0}$.

As in $\S 2$ we next consider a symmetric version. We assume that $\varphi_{\lambda}$ is odd with respect to the space variable: $\varphi_{\lambda}(-x, t)=-\varphi_{\lambda}(x, t)$.

TheOREm 3.2. At least one of the following holds.

(i) There exists a sequence $\left(x_{k}: k \in \mathbf{N}\right)$ of nontrivial stationary points of $\varphi_{\lambda_{0}}$ which converges towards the origin as $k \rightarrow \infty$.

(ii) There exist integers $d_{l}, d_{r} \geq 0$ with $d_{l}+d_{r} \geq\left|m_{l}^{u}-m_{r}^{u}\right|$ such that the following holds: For each $\lambda<\lambda_{0}$ (respectively $\lambda>\lambda_{0}$ ) close to $\lambda_{0}$ there exists a compact set $S_{\lambda} \subset$ $\mathbf{R}^{n}-0$ which is invariant under the flow, symmetric and satisfies genus $\left(S_{\lambda}\right) \geq d_{l}$ (respectively genus $\left.\left(S_{\lambda}\right) \geq d_{r}\right)$. $S_{\lambda}$ contains at least $d_{l}$ (respectively $d_{r}$ ) antipodal pairs $\pm x$ of stationary orbits. Moreover, if there are only finitely many stationary orbits on $S$ then there exist $d_{l}$ (respectively $d_{r}$ ) such stationary pairs $\pm x_{i}$ and connecting orbits from $x_{i+1}$ to $x_{i}$. $S_{\lambda}$ converges towards the origin as $\lambda \rightarrow \lambda_{0}$.

Contrary to the corresponding Theorem 2.4 the direction of the bifurcating solutions is not determined in 3.2(ii). Consider for instance the flow $\varphi_{\lambda}$ associated to the negative gradient vector field $\nabla F-\lambda \nabla G$ where $G(x)=x_{1}^{2}+x_{2}^{2}-x_{3}^{2}$ and $F(x)=x_{1}^{4}+x_{2}^{3} x_{3}+x_{2} x_{3}^{3}$ are as in 2.2c). Since $F$ and $G$ are even $\varphi_{\lambda}$ is odd with respect to the space variable. Here we have supercritical bifurcation, that is, there are nontrivial stationary orbits only for $\lambda>\lambda_{0}=0$. These are $( \pm \sqrt{\lambda / 2}, 0,0)$. If we replace $F$ by $-F$ we obtain subcritical bifurcation branches. Since $F$ contains only terms of higher order than 2 this does not change the dimensions of the unstable manifolds of the origin with respect to $\varphi_{\lambda}$. 
Remark 3.3. As in $\S 2$ we can deal with more general symmetries. We have two types of results in this direction. Suppose a compact Lie group $\Gamma$ acts orthogonally on $\mathbf{R}^{n}$ and the maps $\varphi_{\lambda}$ are equivariant: $\varphi_{\lambda}(\gamma x, t)=\gamma \varphi_{\lambda}(x, t)$ for $\gamma \in \Gamma, x \in \mathbf{R}^{n}$ and $t \in \mathbf{R}$. Let $E_{\lambda}^{u}$ denote the tangent space of the unstable manifold at the origin with respect to the flow $\varphi_{\lambda}$. This is well defined for $\lambda \neq \lambda_{0}$ close to $\lambda_{0}$. Clearly $m_{l}^{u}=\operatorname{dim} E_{\lambda}^{u}$ for $\lambda<\lambda_{0}$ and $m_{r}^{u}=\operatorname{dim} E_{\lambda}^{u}$ for $\lambda>\lambda_{0}$. The equivariance of $\varphi_{\lambda}$ implies that $E_{\lambda}^{u}$ is invariant under the action of $\Gamma$, hence a representation space of $\Gamma$. Its isomorphism class depends only on the sign of $\lambda-\lambda_{0}$. The conclusion of Theorem 3.1 holds under the condition that $E_{\lambda^{-}}^{u}$ and $E_{\lambda^{+}}^{u}$ are not $\Gamma$-homotopy equivalent for $\lambda^{-}<\lambda_{0}<\lambda^{+}$. This is even weaker than the assumption in Remark 2.5 where we required that the corresponding eigenspaces are not stable $\Gamma$-homotopy equivalent.

It is also possible to prove a multiplicity result generalizing Theorem 3.2 to more general symmetries. Its formulation is however more complicated than the corresponding results mentioned in Remark 2.5. One would like that Theorem 3.2 holds true in the $\Gamma$-equivariant case with $m_{l}^{u}-m_{r}^{u}$ replaced by genus $\left(S E_{\lambda^{-}}^{u}\right)-\operatorname{genus}\left(S E_{\lambda^{+}}^{u}\right)$. We did not succeed in proving such a result. Instead we have to replace the genus by a cohomological index theory; cf. $\S 6$. In fact, already in the proof of Theorem 3.2 the length will appear for the group $\Gamma=\mathbf{Z} / 2$ acting on $\mathbf{R}^{n}$ via the reflection at the origin. We would like to mention two particular symmetries which are important in applications. If $\Gamma=\mathbf{Z} / p$ acts without fixed points on $\mathbf{R}^{n}-0$ then Theorem 3.2 remains true verbatim except that antipodal pairs are replaced by $\Gamma$-orbits as in 2.5. Similarly, if $\Gamma=S^{1}$ acts without fixed points on $\mathbf{R}^{n}-0$ then Theorem 3.2 remains true with $m_{l}^{u}-m_{r}^{u}$ replaced by $\left(m_{l}^{u}-m_{r}^{u}\right) / 2$.

4. Nonlinear eigenvalue problems. In this section we apply the results from $\S \S 2,3$ in order to prove some abstract bifurcation theorems for the nonlinear eigenvalue problem

$$
F^{\prime}(x)=\lambda G^{\prime}(x)
$$

Here $F, G: U \rightarrow \mathbf{R}$ are differentiable functions defined on an open neighborhood $U$ of a Hilbert space $X$. Throughout this section we shall assume the following.

$\left(A_{1}\right) F, G \in \mathcal{C}^{2}(U ; \mathbf{R}), F(0)=G(0)=0, F^{\prime}(0)=G^{\prime}(0)=0$.

Thus (4.1) has the trivial solutions $(\lambda, 0) \in \mathbf{R} \times X$. It is possible to parametrize bifurcating nontrivial solutions in two ways. Either we may look for solutions on the level sets $G^{-1}(c)$ with $c$ close to $G(0)=0$ and obtain $\lambda$ as Lagrange multiplier. This corresponds to the situation studied in $\S 2$. Or we may fix a parameter value $\lambda$ near a possible bifurcation value $\lambda_{0}$ and ask for the existence of solutions of (3.1) for this $\lambda$. This leads to the situation investigated in $\S 3$. In order to find nontrivial solutions in the neighborhood of $\left(\lambda_{0}, 0\right)$ we set $L:=F^{\prime \prime}(0)-\lambda_{0} G^{\prime \prime}(0)$ and assume $V:=\operatorname{ker} L \neq 0$. The implicit function theorem shows that this assumption is necessary for bifurcation. Example 2.2a) shows that it is not sufficient.

$\left(A_{2}\right) L=F^{\prime \prime}(0)-\lambda_{0} G^{\prime \prime}(0)$ is a Fredholm operator of index 0.

$\left(A_{3}\right)$ The quadratic form $q: V \rightarrow \mathbf{R}, q(v)=\frac{1}{2}\left\langle G^{\prime \prime}(0) v, v\right\rangle$, is nondegenerate. 
THEOREM 4.2. Suppose $\left(A_{1}\right)-\left(A_{3}\right)$ are satisfied and the signature $\sigma$ of $q$ is not zero. a) At least one of the following holds.

(i) There exists a sequence $\left(\lambda_{k}, x_{k}\right) \in \mathbf{R} \times G^{-1}(0), k \in \mathbf{N}$, of solutions of (4.1) which converges towards $\left(\lambda_{0}, 0\right)$ as $k \rightarrow \infty$.

(ii) For each $c>0$ close to 0 there exists a solution $\left(\lambda_{c}, x_{c}\right) \in \mathbf{R} \times G^{-1}(c)$ of (4.1) which converges towards $\left(\lambda_{0}, 0\right)$ as $c \rightarrow 0$.

(iii) The same statement as in (ii) holds for $c<0$ close to 0.

b) At least one of the following holds.

(i) There exists a sequence $x_{k} \in X-0$ which converges towards 0 , as $k \rightarrow \infty$, such that $\left(\lambda_{0}, x_{k}\right)$ are solutions of $(4.1), k \in \mathbf{N}$.

(ii) For each $\lambda>\lambda_{0}$ close to $\lambda_{0}$ there exists $x_{\lambda} \in X-0$ such that $\left(\lambda, x_{\lambda}\right)$ solves (4.1). Moreover, $x_{\lambda} \rightarrow 0$ as $\lambda \rightarrow \lambda_{0}$.

(iii) The same statement as in (ii) holds for $\lambda<\lambda_{0}$ close to $\lambda_{0}$.

Remark 4.3. a) If $|\sigma|=\operatorname{dim} V$ then there exist at least two solutions $(\lambda, x) \in$ $\mathbf{R} \times G^{-1}(c)$ of (4.1) provided $c \sigma>0$ and $c$ is close to 0 . This has essentially been proved by Böhme [Bö] who considered the case where $G^{\prime}(x)=x$. One can also prove the existence of two solutions parametrized by $\lambda$ if $|\sigma|=\operatorname{dim} V$. A result in this direction is due to Rabinowitz [R1]. It is possible that both solutions bifurcate supercritically or both bifurcate subcritically or that one solution bifurcates subcritically and the other supercritically. The same is true for $|\sigma|=\operatorname{dim} V-2$; observe that $\sigma$ and $\operatorname{dim} V$ have the same parity. If $1 \leq|\sigma| \leq \operatorname{dim} V-4$ we do not know whether there exist two bifurcating branches; cf. the remarks after 2.2. In $\S 2$ we gave examples which show that the direction of the bifurcating solutions (whether (i), (ii) or (iii) holds) is not determined by the terms of second order if $q$ is indefinite.

b) If $\sigma$ is odd then one may apply degree theory methods. These yield locally a continuous branch of solutions, hence (i), (ii) or (iii) must hold in 4.2a), b). Observe however that in order to obtain a global branch additional assumptions on the form of $F^{\prime}-\lambda G^{\prime}$ are necessary. Clearly degree methods do not yield any multiplicity statements without additional "generic" assumptions.

Remark 4.4. Using the same idea as in Example 2.2a) it is not difficult to see that the nonvanishing of the signature is a necessary condition for bifurcation if one doesn't know anything about the higher order terms of $F$ and $G$. More precisely, let $A, B: X \rightarrow X$ be selfadjoint linear operators and set $L:=A-\lambda_{0} B, V:=\operatorname{ker} L$. If the signature of the quadratic form $q(v)=\frac{1}{2}\langle B v, v\rangle$ on $V$ is 0 then there exists a nonlinearity $F(x)=\frac{1}{2}\langle A x, x\rangle+o\left(\|x\|^{2}\right)$ such that the eigenvalue problem $F^{\prime}(x)=\lambda B x$ has no solutions near $\left(\lambda_{0}, 0\right)$ except the trivial ones. Let $V=V^{+} \oplus V^{-}$be a decomposition of $V$ into subspaces $V^{+}$(respectively $V^{-}$) on which $q$ is positive (respectively negative) definit. We write $P^{ \pm}: X \rightarrow V^{ \pm}$for the orthogonal projections and $P:=P^{+}+P^{-}: X \rightarrow V$. Let $i: V^{-} \rightarrow V^{+}$be an isomorphism and set

$$
F(x):=\frac{1}{2}\langle A x, x\rangle+\|P x\|^{2}\left\langle i\left(P^{-} x\right), P^{+} x\right\rangle
$$

It is easy to check that there are no nontrivial solutions of (4.1) for this $F$ and $G(x)=$ $\frac{1}{2}\langle B x, x\rangle$. 
Next we consider the symmetric situation and require the following additional hypothesis.

$\left(A_{4}\right)$ The maps $F$ and $G$ are even: $F(-x)=F(x)$ and $G(-x)=G(x)$.

THEOREM 4.5 Suppose $\left(A_{1}\right)-\left(A_{4}\right)$ are satisfied.

a) If alternative $(i)$ in 4.2a) does not hold then there exist at least $|\sigma|$ antipodal pairs $\left(\lambda_{c}^{i}, \pm x_{c}^{i}\right), i=1, \ldots,|\sigma|$, of solutions of (4.1) on $G^{-1}(c)$ for each $|c|$ small with $c \cdot \sigma>0$. These solutions converge to $\left(\lambda_{0}, 0\right)$ as $c \rightarrow 0$.

b) If alternative ( $i)$ in 4.2b) does not hold then there exist integers $d_{l}, d_{r} \geq 0$ with $d_{l}+d_{r} \geq|\sigma|$ such that $(4.1)$ has at least $d_{l}$ (respectively $d_{r}$ ) antipodal pairs $\left(\lambda, \pm x_{\lambda}^{i}\right)$ of solutions for $\lambda<\lambda_{0}$ (respectively for $\lambda>\lambda_{0}$ ) close to $\lambda_{0}$.

As in $\S \S 2,3$ we can deal with more general symmetry groups; cf. [Ba2], $\S 7$, and [Ba4]. This allows us to replace the assumption $\sigma \neq 0$ in 4.2 by the weaker hypothesis that $V^{+}$ and $V^{-}$are not (stable) $\Gamma$-homotopy equivalent where $V^{ \pm}$are the subspaces of $V$ on which $q$ is positive (negative) definit. For the multiplicity result in 4.5 we have to work with the genus or the length.

Remark 4.6. In the case when $G^{\prime}(x)=x$ and $F$ and $G$ are even functionals Theorem 4.5a) is due to Böhme [Bö] and Marino [Ma] and 4.5b) is due to Fadell and Rabinowitz [FR1]. In [FR2] Fadell and Rabinowitz considered the case $\Gamma=S^{1}$ which was motivated by the search for periodic solutions of autonomous Hamiltonian systems near an equilibrium. Again the bifurcating solutions were parametrized by $\lambda$ which corresponds to the period of the periodic solutions in [FR2]. The case $\Gamma=S^{1}$ has also been considered in [Ba3] where Theorem 4.5a) is proved implicitely in a special situation. The paper [Ba3] was motivated by the search for periodic solutions of Hamiltonian systems lying on fixed energy surfaces.

For the proofs of Theorems 4.2 and 4.5 we make a finite-dimensional reduction as follows. First we apply the Lyapunov-Schmidt reduction to equation $(4.1)$ near $\left(\lambda_{0}, 0\right)$. This yields a $\mathcal{C}^{1}$-map

$$
w^{*}: U\left(\lambda_{0}, 0\right) \rightarrow W:=V^{\perp}
$$

where $U\left(\lambda_{0}, 0\right)$ is a neighborhood of $\left(\lambda_{0}, 0\right)$ in $\mathbf{R} \times V$ with the following property. The equation

$$
(I-P) \circ\left(F^{\prime}(x)-\lambda G^{\prime}(x)\right)=0
$$

is satisfied for $x=v+w \in X=V \oplus W$ near 0 and $\lambda$ near $\lambda_{0}$ if, and only if, $w=w^{*}(\lambda, v)$. Moreover, if $F$ and $G$ are even maps then $F^{\prime}$ and $G^{\prime}$ are odd and $w^{*}$ is odd: $w^{*}(\lambda,-v)=$ $-w^{*}(\lambda, v)$. Thus it remains to solve the bifurcation equation

$$
P \circ\left(F^{\prime}\left(v+w^{*}(\lambda, v)\right)-\lambda G^{\prime}\left(v+w^{*}(\lambda, v)\right)\right)=0
$$

The left hand side of (4.7) defines a $\mathcal{C}^{1}$-vector field near 0 in $V$, parametrized by $\lambda \in \mathbf{R}$. Let $\varphi_{\lambda}$ be the associated local flow in $U\left(\lambda_{0}, 0\right)$. It is not difficult to check that the map

$$
v \mapsto F\left(v+w^{*}(\lambda, v)\right)-\lambda G\left(v+w^{*}(\lambda, v)\right)
$$


is a Lyapunov function for $\varphi_{\lambda}$, so $\varphi_{\lambda}$ is gradient-like. Let $L_{\lambda}: V \rightarrow V$ be the linearization of the vector field at 0 . A simple calculation shows

$$
L_{\lambda}=P \circ\left(F^{\prime \prime}(0)-\lambda G^{\prime \prime}(0)\right) \circ i=\left(\lambda-\lambda_{0}\right) P \circ G^{\prime \prime}(0) \circ i
$$

where $i: V \rightarrow X$ denotes the inclusion. By hypothesis $\left(A_{3}\right)$ we may split $V$ as $V \cong V^{+} \oplus$ $V^{-}$such that $V^{+}$is the eigenspace of $P \circ G^{\prime \prime}(0) \circ i$ corresponding to the positive eigenvalues and $V^{-}$the eigenspace corresponding to the negative eigenvalues. Consequently, 0 is a hyperbolic fixed point of $\varphi_{\lambda}$ for $\lambda \neq \lambda_{0}$ and the dimension of the unstable manifold is $m_{l}^{u}=\operatorname{dim} V^{+}$for $\lambda<\lambda_{0}$, respectively $m_{r}^{u}=\operatorname{dim} V^{-}$for $\lambda>\lambda_{0}$. Thus $m_{l}^{u}-m_{r}^{u}=\sigma$, the signature of the quadratic form induced by $G^{\prime \prime}(0)$ on $V$. Now 4.2b) follows from 3.1 and 4.5b) from 3.2.

For the proofs of $4.2 \mathrm{a}$ ) and $4.5 \mathrm{a}$ ) we have to rewrite (4.7) as a constrained variational problem. Taking the inner product of (4.7) with $v^{+}-v^{-}$yields the equation

$$
\left\langle\left(F^{\prime}-\lambda G^{\prime}-L\right)\left(v+w^{*}(\lambda, v)\right), v^{+}-v^{-}\right\rangle=0
$$

where we also used that the image of $L$ is $W$ which is orthogonal to $V$. Now we set

$$
g(\lambda, v):=\lambda-\lambda_{0}-\frac{\left\langle\left(F^{\prime}-\lambda_{0} G^{\prime}-L\right)\left(v+w^{*}(\lambda, v)\right), v^{+}-v^{-}\right\rangle}{\left\langle G^{\prime}\left(v+w^{*}(\lambda, v)\right), v^{+}-v^{-}\right\rangle} \quad \text { for } v \neq 0
$$

and

$$
g(\lambda, v):=\lambda-\lambda_{0} \quad \text { for } v=0 .
$$

Clearly equation (4.8) is equivalent to $g(\lambda, v)=0$ for $v \neq 0$. As in the proof of Lemma 6.11 of $[\mathrm{MW}]$ one checks that $g: U\left(\lambda_{0}, 0\right) \rightarrow \mathbf{R}$ is well defined and continuous. Moreover, $\frac{\partial g}{\partial \lambda}(\lambda, v)$ exists for all $(\lambda, v) \in U\left(\lambda_{0}, 0\right)$ and is continuous with $\frac{\partial g}{\partial \lambda}\left(\lambda_{0}, 0\right)=1$. In addition, $\frac{\partial g}{\partial v}(\lambda, v)$ exists for $v \neq 0$ and is continuous. The implicit function theorem yields a continuous map

$$
\lambda^{*}: U(0) \rightarrow \mathbf{R}
$$

where $U(0) \subset V$ is a neighborhood of 0 in $V$, with the following properties. The equation $g(\lambda, v)=0$ is satisfied near $\left(\lambda_{0}, 0\right)$ iff $\lambda=\lambda^{*}(v)$. In particular, $\lambda^{*}(0)=\lambda_{0}$. The map $\lambda^{*}$ is of class $\mathcal{C}^{1}$ in $U(0)-\{0\}$.

Therefore, setting $\bar{w}(v):=w^{*}\left(\lambda^{*}(v), v\right)$ it remains to solve

$$
P\left(F^{\prime}(v+\bar{w}(v))-\lambda^{*}(v) G^{\prime}(v+\bar{w}(v))\right)=0 .
$$

This is defined for $v \in U(0) \subset V$. The equivariance of $w^{*}$ implies that $g$ is even, hence $\lambda^{*}$ is even and $\bar{w}$ is odd. Next we define the functions

$$
F_{0}: U(0) \rightarrow \mathbf{R}, v \mapsto F(v+\bar{w}(v)),
$$

and

$$
G_{0}: U(0) \rightarrow \mathbf{R}, v \mapsto G(v+\bar{w}(v))
$$

These functionals are of class $\mathcal{C}^{1}$ and even. Moreover, $G_{0}(0)=0$ and $G_{0}^{\prime}(0)=0$. One can check that $G_{0}^{\prime \prime}(0)$ exists and is given by $G_{0}^{\prime \prime}(0)=P \circ G^{\prime \prime}(0) \circ i$. Consequently, $G_{0}^{\prime \prime}(0)$ is nondegenerate. This suffices to apply the Morse lemma to $G_{0}$. After a $\mathcal{C}^{1}$-change of coordinates we may assume that $G_{0}\left(v^{+}+v^{-}\right)=\left\|v^{+}\right\|^{2}-\left\|v^{-}\right\|^{2}$. If $F_{0}$ would be of class $\mathcal{C}^{2}$ in these new coordinates then we could integrate the vector field $\nabla_{G_{0}} F_{0}$ and apply 
Theorems 2.1 and 2.4 to the associated flow $\varphi$. Since this is not the case we have to replace $\nabla_{G_{0}} F_{0}$ by a locally Lipschitz vector field which leaves the level sets of $G_{0}$ invariant and has $F_{0}$ as a Lyapunov function. Of course, the zeroes of the new vector field should be close to the zeroes of $\nabla_{G_{0}} F_{0}$. The technical details are carried out in [Ba4], a special case has been considered in [Ba3].

5. Conley index, genus and length. In this section we first recall basic results from Conley index theory. We assume the reader to be familiar with the standard version of Conley index theory as developed for instance in $[\mathrm{Co}]$, $[\mathrm{CoZ}]$ or $[\mathrm{Sa}]$. We just introduce the basic notions without proofs.

Let $M$ be a locally compact metric space on which the group $\mathbf{Z} / 2$ acts continuously (a $\mathbf{Z} / 2$-space). This just means that we are given a continuous involution $I: M \rightarrow M$. Subsets $A$ of $M$ with $I(A)=A$ are called symmetric. Let $\varphi$ be a continuous local flow on $M$ which is equivariant, that is, $\varphi(I x, t)=I \varphi(x, t)$. A compact subset $S$ of $M$ is said to be isolated invariant if there exists a neighborhood $N$ of $S$ in $M$ with

$$
S=\operatorname{inv}(N):=\left\{x \in M: \varphi^{t}(x) \in N \text { for all } t \in \mathbf{R}\right\} .
$$

In particular, $S$ is invariant with respect to the flow: $\varphi^{t}(x)$ is defined for all $x \in S$ and $t \in \mathbf{R}$ and lies in $S$. A neighborhood $N$ as above is called an isolating neighborhood of $S$.

An index pair for an isolated invariant set $S$ is a pair $(N, A)$ of compact $\mathbf{Z} / 2$-invariant subsets $A \subset N$ of $M$ with:

- $N-A$ is an isolating neighborhood of $S$;

- $A$ is positively invariant with respect to $N$, that is, if $x \in A$ and $\varphi^{t}(x) \in N$ for all $0 \leq t \leq t_{0}$ then $\varphi^{t}(x) \in A$ for $0 \leq t \leq t_{0}$;

- $A$ is an exit set for $N$, that is, if $x \in N$ and $\varphi^{t_{0}}(x) \notin N$ for some $t_{0}>0$ then there exists $t \in\left[0, t_{0}\right]$ with $\varphi^{t}(x) \in A$.

An index pair $(N, A)$ is said to be regular if the map

$$
\tau: N \rightarrow[0, \infty], \tau(x):= \begin{cases}\sup \{t>0: \varphi(x \times[0, t]) \subset N-A\} & \text { if } x \in N-A \\ 0 & \text { if } x \in A\end{cases}
$$

is continuous. It is obviously invariant (that is, $\tau(I x)=\tau(x)$ for $x \in N$ ) since $\varphi$ is equivariant. The starting point of Conley index theory is the following fact. For any neighborhood $U$ of an isolated invariant and symmetric subset $S$ of $M$ there exists a symmetric index pair $(N, A)$ for $S$ contained in U. If $(N, A)$ and $\left(N^{\prime}, A^{\prime}\right)$ are two such index pairs for $S$ then the quotient spaces $N / A$ and $N^{\prime} / A^{\prime}$ are homotopy equivalent as $\mathbf{Z} / 2$-spaces with base points. The Conley index $\mathcal{C}(S)$ of an isolated invariant set $S$ is the based $\mathbf{Z} / 2$-homotopy type of $N / A$ where $(N, A)$ is an index pair for $S$. We use the convention $N / \emptyset:=N \sqcup$ pt.

An important property of the Conley index is its invariance under continuation. For us the following version of the continuation invariance suffices. Let $\varphi_{\lambda}$ be a family of (local) flows defined on $M$, continuously parametrized by $\lambda \in \Lambda$. Let $N \subset M$ be an isolating neighborhood for $\varphi_{\lambda_{0}}$. Then $N$ is also isolating for $\varphi_{\lambda}$ if $\lambda$ is close to $\lambda_{0}$. Setting $S_{\lambda}:=$ 
$\operatorname{inv}\left(N, \varphi_{\lambda}\right)$ the continuation invariance now means that the Conley indices $\mathcal{C}\left(S_{\lambda}, \varphi_{\lambda}\right)$ are independent of $\lambda$ (in a neighborhood of $\lambda_{0}$ ).

For the proofs of the results of $\S \S 2,3$ we combine the Conley index with some ideas from minimax theory. We first need the notion of index theory (for the group $\mathbf{Z} / 2$ ).

Definition 5.1. An index theory $i$ associates to a $\mathbf{Z} / 2$-space $A$ an element $i(A) \in$ $\mathbf{N} \cup\{\infty\}$ with the following properties.

a) Monotonicity: If there exists an equivariant map $A \rightarrow A^{\prime}$ then $i(A) \leq i\left(A^{\prime}\right)$.

b) Subadditivity: If $A$ and $A^{\prime}$ are open subsets of $A \cup A^{\prime}$ then $i\left(A \cup A^{\prime}\right) \leq i(A)+i\left(A^{\prime}\right)$.

c) Continuity: Any locally closed invariant subset $B$ of a metrizable $\mathbf{Z} / 2$-space $A$ has an invariant neighborhood $N$ with $i(N)=i(B)$. Here $B$ is said to be locally closed if it is the intersection of a closed and an open subset of $A$.

d) Strong Normalization: $i(A)=1$ for every finite $\mathbf{Z} / 2$-space without fixed points.

It is well known that the genus has all these properties. It has the additional property that $i\left(S^{n-1}\right)=n$ where $\mathbf{Z} / 2$ acts on $S^{n-1}$ via the antipodal map. A simple consequence of the properties of an index theory is the following result whose proof can be found in [Ba2], Theorem 6.3.

Proposition 5.2. Let $S \subset M$ be a compact invariant symmetric set without fixed points of the action. Assume moreover that the flow $\varphi$ on $S$ is gradient-like. Then there exist at least $i(S)$ different $\mathbf{Z} / 2$-orbits of stationary points on $S$. If there are only finitely many stationary $\mathbf{Z} / 2$-orbits on $S$ then there exist $i(S)$ such orbits $\left\{x_{j}, I x_{j}\right\}$ and connecting orbits from $\left\{x_{j+1}, I x_{j+1}\right\}$ to $\left\{x_{j}, I x_{j}\right\}$.

For the proof of Theorem 3.2 we need a special index theory which has more properties. Moreover, we want it to be defined for pairs $(A, B)$ of $\mathbf{Z} / 2$-spaces. In order to define it we need to recall Borel cohomology; see [tD] for its basic properties. Let $H^{*}\left(-; \mathbf{F}_{2}\right)$ denote Alexander-Spanier or Čech cohomology (cf. [D] or [Sp]) with coefficients in the field $\mathbf{F}_{2}$ of two elements. Let $\mathrm{E} \Gamma$ be a contractible space with a free action of $\Gamma=\mathbf{Z} / 2$; for example we may take the unit sphere of an infinite-dimensional normed vector space where $\Gamma$ acts via the antipodal map. $\mathrm{E} \Gamma$ is determined up to equivariant homotopy. For $\Gamma$-spaces $B \subset A$ we write

$$
h^{*}(A, B):=H^{*}\left((\mathrm{E} \Gamma \times A) / \Gamma,(\mathrm{E} \Gamma \times B) / \Gamma ; \mathbf{F}_{2}\right)
$$

for the Borel cohomology of $(A, B)$. Here $(\mathrm{E} \Gamma \times A) / \Gamma$ denotes the orbit space of the diagonal actional of $\Gamma$ on $\mathrm{E} \Gamma \times A$. Observe that $\mathrm{E} \Gamma / \Gamma=\mathrm{B} \Gamma$ is the classifying space of $\Gamma$. It is unique up to homotopy and homotopy equivalent to $\mathbf{R} P^{\infty}$. Therefore the coefficient ring is

$$
R:=h^{*}(\mathrm{pt}) \cong H^{*}\left(\mathrm{~B} \Gamma ; \mathbf{F}_{2}\right) \cong \mathbf{F}_{2}[w]
$$

with a generator $w \in H^{1}\left(\mathrm{~B} \Gamma ; \mathbf{F}_{2}\right) \cong \mathbf{F}_{2}$. The cup product in cohomology turns $h^{*}(A)$ into a graded commutative ring with unit and $h^{*}(A, B)$ into a module over $h^{*}(A)$. The homomorphism $R \rightarrow h^{*}(A)$ induced by $A \rightarrow$ pt induces an $R$-module structure on each $h^{*}(A, B)$. 
Definition 5.3. For a pair $(A, B)$ of $\mathbf{Z} / 2$-spaces the length $\ell(A, B)$ of $(A, B)$ is defined to be

$$
\begin{aligned}
\ell(A, B) & :=\min \left\{k \in \mathbf{N}: w^{k} \in R \text { annihilates } h^{*}(A, B)\right\} \\
& =\min \left\{k \in \mathbf{N}: w^{k} \xi=0 \text { for all } \xi \in h^{*}(A, B)\right\} \\
& =1+\max \left\{k \in \mathbf{N}: w^{k} \xi \neq 0 \text { for some } \xi \in h^{*}(A, B)\right\} .
\end{aligned}
$$

We use the convention $\min \emptyset=\infty$.

This notion is due to Yang $[\mathrm{Y}]$, at least if $B=\emptyset$. Yang calls it cohomological index. It has been used by Fadell and Rabinowitz [FR1] in their proof of 4.5b).

Proposition 5.4. In addition to the properties of an index theory the length $\ell$ has the following properties.

a) $\ell(A, B) \leq \ell(A)$ for any invariant subspace $B$ of $A$.

b) Triangle inequality: For any triple $C \subset B \subset A$ of $\mathbf{Z} / 2$-spaces

$$
\ell(A, B)+\ell(B, C) \geq \ell(A, C) .
$$

c) If $C \subset B \subset A$ are invariant subspaces and $C$ is closed in $A$ then

$$
\ell(A, B)=\ell(A / C, B / C) \text {. }
$$

Proof. The statements follow easily from the properties of $h^{*}$. See [FR1] for the proof that $\ell$ is an index theory or [Ba2], §4.4.

6. Proof of Theorems 2.1 and 2.4. For the proof of Theorem 2.1 we may assume that $U$ is an open ball in $V=\mathbf{R}^{n}$ and $G(y, z)=\|z\|^{2}-\|y\|^{2}$ with $(y, z) \in U \subset V=$ $V^{-} \oplus V^{+}$. Here $\operatorname{dim} V^{-}=\mu$ and $\operatorname{dim} V^{+}=n-\mu$. This is an easy consequence of the Morse lemma. We shall write $\Sigma_{c}:=G^{-1}(c)$ and $\varphi_{c}$ for the local flow $\varphi$ restricted to $\Sigma_{c}$. Clearly $\Sigma_{c} \cong S V^{-} \times D V^{+} \cong S^{\mu-1} \times D^{n-\mu}$ for $c<0$ and $\Sigma_{c} \cong D V^{-} \times S V^{+} \cong D^{\mu} \times S^{n-\mu-1}$ for $c>0$. If 2.1(i) does not hold then $S_{0}:=\{0\} \subset \Sigma_{0}$ is an isolated invariant set for $\varphi_{0}$ on $\Sigma_{0}$. We choose $\varepsilon>0$ so that $S_{0}=\operatorname{inv}\left(\Sigma_{0} \cap B_{\varepsilon}(0), \varphi_{0}\right)$. Then $B_{\varepsilon}(0)$ is also an isolating neighborhood for $\varphi_{c}$ provided $|c| \leq c_{0}$ is small. We define

$$
S_{c}:=\operatorname{inv}\left(\Sigma_{c} \cap B_{\varepsilon}(0), \varphi_{0}\right) \subset \Sigma_{c}
$$

and

$$
S:=\bigcup_{|c| \leq c_{0}} S_{c} \subset \Sigma:=\bigcup_{|c| \leq c_{0}} \Sigma_{c}
$$

We cannot apply the continuation invariance of the Conley index to the map $c \mapsto$ $\mathcal{C}\left(S_{c}, \varphi_{c}\right)$ because the local flows $\varphi_{c}$ are defined on different spaces depending on the $\operatorname{sign}$ of $c$. However, this map is constant on $-c_{0} \leq c<0$ and on $0<c \leq c_{0}$. Now one can construct an index pair $(N, A)$ for $S$ in $\Sigma$ with the following additional properties:

- $S \subset Q:=N \cap U_{\delta}(0)$ where $\delta>0$ is small

- $A \subset P:=N-\bar{Q}$

- $A_{c}:=A \cap \Sigma_{c}$ is independent of $c$ (up to homeomorphisms) for $|c|$ small

- $P_{c}:=P \cap \Sigma_{c}$ is independent of $c$ for $|c|$ small 
This implies that $Q_{c} \cap\left(P_{c} / A_{c}\right) \cong S V^{-} \times S V^{+}$is also independent of $c$. On the other hand, $N_{c}:=N \cap \Sigma_{c}$ and $Q_{c}$ are not independent of $c$ because

$$
Q_{c} \cong \begin{cases}S V^{-} \times D V^{+} & \text {if } c<0 \\ D V^{-} \times S V^{+} & \text {if } c>0 \\ S V^{-} \times D V^{+} / S V^{-} \times\{0\} & \text { if } c=0\end{cases}
$$

Next we look at the Mayer-Vietoris sequence of the triad $\left(N_{c} / A_{c} ; P_{c} / A_{c}, Q_{c}\right)$.

$\ldots \rightarrow \tilde{H}_{*+1}\left(N_{c} / A_{c}\right) \rightarrow \tilde{H}_{*}\left(Q_{c} \cap\left(P_{c} / A_{c}\right)\right) \rightarrow \tilde{H}_{*}\left(Q_{c}\right) \oplus \tilde{H}_{*}\left(P_{c} / A_{c}\right) \rightarrow \tilde{H}_{*}\left(N_{c} / A_{c}\right) \rightarrow \ldots$

If 2.1(ii) does not hold then there exists a sequence $c_{k} \rightarrow 0$ such that $S_{c_{k}}=\emptyset$, hence $N_{c_{k}} / A_{c_{k}} \simeq$ pt. This implies for $c>0$ small:

$$
\tilde{H}_{*}\left(S V^{-} \times S V^{+}\right) \cong \tilde{H}_{*}\left(Q_{c} \cap\left(P_{c} / A_{c}\right)\right) \cong \tilde{H}_{*}\left(Q_{c}\right) \oplus \tilde{H}_{*}\left(P_{c} / A_{c}\right)
$$

Since $\tilde{H}_{*}\left(P_{c} / A_{c}\right)$ is independent of $c$ and $\tilde{H}_{*}\left(Q_{c}\right)$ changes as $c$ passes 0 it follows that $\tilde{H}_{*}\left(\mathcal{C}\left(S_{c}, \varphi_{c}\right)\right)=\tilde{H}_{*}\left(N_{c} / A_{c}\right) \neq 0$ for $c<0$ close to 0 . This implies $S_{c} \neq \emptyset$ for $c<0$ close to 0 , hence, there exists a stationary solution on $\Sigma_{c}$. This proves Theorem 2.1.

We come to the proof of Theorem 2.4. Suppose 2.4(i) does not hold. We may assume that $d:=n-2 \mu>0$; the case $d<0$ can be treated similarly. Observe that $d=$ $\operatorname{genus}\left(S V^{+}\right)-\operatorname{genus}\left(S V^{-}\right)$. Since 2.4(i) does not hold we can define as above the isolated invariant sets

$$
S_{c}=\operatorname{inv}\left(\Sigma_{c} \cap B_{\varepsilon}(0), \varphi_{c}\right) \subset \Sigma_{c}
$$

We claim that genus $\left(S_{c}\right) \geq d$ for $c>0$. Then Theorem 2.4 follows immediately from Proposition 5.2. In order to see this inequality we choose a regular $G$-index pair $\left(N_{c}, A_{c}\right)$ of $S_{c}$. Observing that $N_{c} \cap\left(\{0\} \times V^{+}\right) \cong S V^{+}$if $c>0$ is small we obtain $\operatorname{genus}\left(N_{c}\right) \geq$ genus $\left(S V^{+}\right)$by the monotonicity of the genus. In fact we have equality because $N_{c} \subset$ $\Sigma_{c} \cong V^{-} \times S V^{+}$. Moreover, since $A_{c} \subset \Sigma_{c}-\left(\{0\} \times V^{+}\right) \simeq S V^{-} \times S V^{+}$we get $\operatorname{genus}\left(A_{c}\right) \leq$ $\operatorname{genus}\left(S V^{-} \times S V^{+}\right) \leq \operatorname{genus}\left(S V^{-}\right)$, again by the monotonicity property of the genus. Recall the continuous map $\tau: N_{c} \rightarrow[0, \infty]$ from the definition of a regular index pair. The set $B_{c}:=\tau^{-1}([0, \infty)) \subset N_{c}$ is open and the map

$$
B_{c} \rightarrow A_{c}, v \mapsto \varphi_{c}(v, \tau(v)),
$$

is equivariant. Hence genus $\left(B_{c}\right) \leq \operatorname{genus}\left(A_{c}\right)$. Next we claim that for $D_{c}:=N_{c}-B_{c}$ we have genus $\left(D_{c}\right) \leq \operatorname{genus}\left(S_{c}\right)$. In order to see this we choose a neighborhood $U\left(S_{c}\right)$ of $S_{c}$ with genus $\left(U\left(S_{c}\right)\right)=\operatorname{genus}\left(S_{c}\right)$, which exists due to the continuity property of the genus. For $v \in D_{c}$ we have $\tau(v)=\infty$, hence $\omega(v) \subset S_{c}$ holds for the $\omega$-limit set of $v$. This implies that there exists $t(v)>0$ such that $\varphi(v, t) \in U\left(S_{c}\right)$ for all $t \geq t(v)$. Therefore we have an equivariant map

$$
D_{c} \rightarrow U\left(S_{c}\right), v \mapsto \varphi(v, T(v)),
$$

where $T(v)$ is constructed from $t(v)$ via a partition of unity. This implies genus $\left(D_{c}\right) \leq$ $\operatorname{genus}\left(U\left(S_{c}\right)\right)=\operatorname{genus}\left(S_{c}\right)$. Now we can apply the subadditivity property of the genus 
and obtain

$$
\begin{aligned}
\operatorname{genus}\left(S V^{+}\right) & \leq \operatorname{genus}\left(N_{c}\right) \\
& \leq \operatorname{genus}\left(B_{c}\right)+\operatorname{genus}\left(D_{c}\right) \\
& \leq \operatorname{genus}\left(A_{c}\right)+\operatorname{genus}\left(S_{c}\right) \\
& \leq \operatorname{genus}\left(S V^{-}\right)+\operatorname{genus}\left(S_{c}\right)
\end{aligned}
$$

This yields the desired inequality $\operatorname{genus}\left(S_{c}\right) \geq d=\operatorname{genus}\left(S V^{+}\right)-\operatorname{genus}\left(S V^{-}\right)$.

7. Proof of Theorems 3.1 and 3.2. We begin with the proof of Theorem 3.1 and assume first that 3.1(i) does not apply. Then $S_{\lambda_{0}}:=\{0\} \subset \mathbf{R}^{n}$ is an isolated invariant set for $\varphi_{\lambda_{0}}$. We choose $\varepsilon>0$ so that $S_{\lambda_{0}}=\operatorname{inv}\left(B_{\varepsilon}(0), \varphi_{\lambda_{0}}\right)$. Then $B_{\varepsilon}(0)$ is also an isolating neighborhood for $\varphi_{\lambda}$ provided $\lambda$ is close to $\lambda_{0}$. We define

$$
S_{\lambda}:=\operatorname{inv}\left(B_{\varepsilon}(0), \varphi_{\lambda}\right) \subset \mathbf{R}^{n}
$$

The continuation invariance of the Conley index implies that $\mathcal{C}\left(S_{\lambda}, \varphi_{\lambda}\right)$ is independent of $\lambda$ near $\lambda_{0}$. If also 3.1(ii) does not hold then there exists a sequence $\lambda_{k}>\lambda_{0}$ converging to $\lambda_{0}$ such that $S_{\lambda_{k}}=\{0\}$. This implies for $\lambda$ close to $\lambda_{0}$ :

$$
\mathcal{C}\left(S_{\lambda}, \varphi_{\lambda}\right)=\mathcal{C}\left(S_{\lambda_{k}}, \varphi_{\lambda_{k}}\right)=\left[S^{m_{r}^{u}}\right]
$$

where [ ] denotes the pointed homotopy type and $m_{r}^{u}$ is the dimension of the unstable manifold of 0 with respect to $\varphi_{\lambda}$ for $\lambda>\lambda_{0}$. For $\lambda<\lambda_{0}$ we have correspondingly $\mathcal{C}\left(\{0\}, \varphi_{\lambda}\right)=\left[S^{m_{l}^{u}}\right]$. By assumption we have $m_{l}^{u} \neq m_{r}^{u}$ hence $S_{\lambda} \neq\{0\}$ for $\lambda<\lambda_{0}$ close to $\lambda_{0}$. This implies that $S_{\lambda}$ must contain another stationary solution because the flow is gradient-like. That these solutions converge towards the origin as $\lambda \rightarrow \lambda_{0}$ is clear since they lie in $B_{\varepsilon}(0)$ and $\varepsilon>0$ may be chosen as small as we please. This proves Theorem 3.1 .

The proof of Theorem 3.2 is much more complicated. A complete proof even for more general symmetries can be found in [Ba2], $\$ 7.5$. We shall only give a sketch of the proof in order to illustrate the analogy and the differences to the proof of Theorem 2.4. This seems to be useful since in [Ba2] more general symmetries have been considered. The special $\mathbf{Z} / 2$-symmetry allows a somewhat easier argument but contains the essential points. We have to work with the length defined in 5.3. We begin as usual. Assuming that 3.2(i) does not hold we choose $\varepsilon>0$ small and set

$$
S_{\lambda}^{\prime}:=\operatorname{inv}\left(B_{\varepsilon}(0), \varphi_{\lambda}\right) \subset \mathbf{R}^{n}
$$

Then $0 \in S_{\lambda}^{\prime}$ for every $\lambda$ and $S_{\lambda_{0}}^{\prime}=\{0\}$. This implies that $i\left(S_{\lambda}^{\prime}\right)=\infty$ for $i=$ genus or $i=\ell$. We look at the lengths of the unstable sets of 0 and of $S_{\lambda}^{\prime}$, respectively. By this we mean the following. For any isolated invariant set $S$ of a flow $\varphi$ and any isolating neighborhood $N$ of $S$ we set

$$
\begin{aligned}
N^{u} & =\left\{x \in N-S: \varphi^{t}(x) \in N \text { for all } t \leq 0\right\} \\
& =\{x \in N-S: \alpha(x) \subset S\}
\end{aligned}
$$

If $N^{\prime}$ is another isolating neighborhood of $S$ then there exists a real number $t \leq 0$ such that $\varphi^{t}\left(N^{u}\right) \subset N^{\prime u}$ and $\varphi^{t}\left(N^{\prime u}\right) \subset N^{u}$. By the monotonicity property the number $\ell^{u}(S, \varphi):=$ 
$\ell\left(N^{u}\right)$ is well defined, i.e. independent of the choice of an isolating neighborhood $N$ of $S$. It is called the exit-length of $S$. We need two properties of the exit-length.

Proposition 7.1. $\ell^{u}(S)=\min \{\ell(A):(N, A)$ is an index pair for $S\}$.

This result is true in general whereas the following one works only in our special situation. It uses the fact that $0 \in S_{\lambda}^{\prime}$.

Proposition 7.2. The exit-length $\ell^{u}\left(S_{\lambda}^{\prime}, \varphi_{\lambda}\right)$ is independent of $\lambda$ near $\lambda_{0}$.

For the proofs of 7.1 and 7.2 see [Ba2], Proposition 7.3, Theorem 7.4. In the definition of the exit-length we only needed the monotonicity of $\ell$. Thus we can also define the exitindex $i^{u}(S, \varphi)=i\left(N^{u}\right)$ where $i$ is any index theory. It seems however that the propositions 7.1 and 7.2 are only true for the length. In addition to the properties of $\ell$ listed in 5.4 one needs still more properties which heavily depend on properties of cohomology theories. The basic idea of the proof of Theorem 3.2 is easy. By 7.2 the exit-length $\ell^{u}\left(S_{\lambda}^{\prime}, \varphi_{\lambda}\right)$ remains invariant but the exit-length $\ell^{u}\left(\{0\}, \varphi_{\lambda}\right)$ changes as $\lambda$ passes $\lambda_{0}$. More precisely, we have

$$
\ell^{u}\left(\{0\}, \varphi_{\lambda}\right)= \begin{cases}m_{l}^{u} & \text { if } \lambda<\lambda_{0} \\ m_{r}^{u} & \text { if } \lambda>\lambda_{0}\end{cases}
$$

We define $d_{l}:=\left|\ell^{u}\left(\{0\}, \varphi_{\lambda_{0}}\right)-m_{l}^{u}\right|$ and $d_{r}:=\left|\ell^{u}\left(\{0\}, \varphi_{\lambda_{0}}\right)-m_{r}^{u}\right|$. Clearly we have $d_{l}+d_{r} \geq\left|m_{l}^{u}-m_{r}^{u}\right|$. We claim that there exists an invariant subset $S_{\lambda} \subset S_{\lambda}^{\prime}-\{0\}$ with $\ell\left(S_{\lambda}\right) \geq d_{l}$ for $\lambda<\lambda_{0}$ and $\ell\left(S_{\lambda}\right) \geq d_{r}$ for $\lambda>\lambda_{0}$ close to $\lambda_{0}$. We fix some $\lambda<\lambda_{0}$ and assume first that $\ell^{u}\left(S_{\lambda}^{\prime}, \varphi_{\lambda}\right)=\ell^{u}\left(\{0\}, \varphi_{\lambda_{0}}\right)<m_{l}^{u}$. By Proposition 7.1 we may choose an index pair $\left(N_{1}, A_{1}\right)$ of $S_{\lambda}^{\prime}$ contained in $B_{\varepsilon}(0)$ such that $\ell\left(A_{1}\right)=\ell^{u}\left(S_{\lambda}^{\prime}, \varphi_{\lambda}\right)$. Similarly we may choose an index pair $\left(N_{2}, A_{2}\right)$ of 0 with $\ell\left(A_{2}\right)=\ell^{u}\left(\{0\}, \varphi_{\lambda}\right)=m_{l}^{u}$. Now we define

$$
B:=\left\{x \in A_{2}: \omega(x) \subset S_{\lambda}^{\prime}\right\}
$$

and

$$
S_{\lambda}:=\omega(B) \subset S_{\lambda}^{\prime}-\{0\}
$$

$B$ and $S_{\lambda}$ are closed symmetric subsets of $B_{\varepsilon}(0)$ and $S_{\lambda}$ is invariant. A point $x \in A_{2}-B$ must eventually leave $B_{\varepsilon}(0)$, hence it must leave $N_{1}$, because otherwise its $\omega$-limit set would be contained in $S_{\lambda}^{\prime}$ which is not possible. Using the monotonicity property of the length it follows that

$$
\ell\left(A_{2}-B\right) \leq \ell^{u}\left(S_{\lambda}^{\prime}, \varphi_{\lambda}\right)=\ell^{u}\left(\{0\}, \varphi_{\lambda_{0}}\right)
$$

Using the continuity and the subadditivity property of the length we obtain

$$
m_{l}^{u}=\ell\left(A_{2}\right) \leq \ell(B)+\ell\left(A_{2}-B\right) \leq \ell(B)+\ell^{u}\left(\{0\}, \varphi_{\lambda_{0}}\right)
$$

Finally, $\ell\left(S_{\lambda}\right) \geq \ell(B)$ because $S_{\lambda}=\omega(B)$; here we use the monotonicity and continuity of the length once more. Putting these inequalities together we obtain

$$
\ell\left(S_{\lambda}\right) \geq m_{l}^{u}-\ell^{u}\left(\{0\}, \varphi_{\lambda_{0}}\right)=d_{l}
$$

It remains to consider the case $\ell^{u}\left(S_{\lambda}^{\prime}, \varphi_{\lambda}\right)>m_{l}^{u}$. This can be reduced to the first case by replacing the flow $\varphi_{\lambda}$ by its inverse flow $\varphi_{\lambda}^{-}$where $\varphi_{\lambda}^{-}(x, t):=\varphi_{\lambda}(x,-t)$. We need the following computations for this reduction. 
Proposition 7.3. a) $\ell^{u}\left(\{0\}, \varphi_{\lambda_{0}}^{-}\right)=n-\ell^{u}\left(\{0\}, \varphi_{\lambda_{0}}\right)$

b) $\ell^{u}\left(S_{\lambda}^{\prime}, \varphi_{\lambda}^{-}\right)=n-\ell^{u}\left(S_{\lambda}^{\prime}, \varphi_{\lambda}\right)$.

This is a kind of duality for the exit-length. A proof of part a) can be found in [Ba2], Proposition 7.7. Part b) can be proved similarly. It would be interesting to investigate whether a similar duality holds for the exit-length in more general situations.

Arguing as in the first case we obtain a compact invariant set $S_{\lambda}$ with

$$
\ell\left(S_{\lambda}\right) \geq\left(n-m_{l}^{u}\right)-\ell^{u}\left(\{0\}, \varphi_{\lambda_{0}}^{-}\right)=\ell^{u}\left(\{0\}, \varphi_{\lambda_{0}}\right)-m_{l}^{u}=d_{l}
$$

This finishes the proof of Theorem 3.2.

Comparing the proofs in this section with those in $\S 6$ it is interesting to observe that the proof of Theorem 3.1 is simpler than the one of the corresponding Theorem 2.1 whereas the proof of Theorem 3.2 is more complicated than the one of Theorem 2.4. The propositions $7.1-7.3$ are not trivial.

\section{References}

[Ba1] T. Bartsch, On the genus of representation spheres, Comment. Math. Helv. 65 (1990), 85-95.

[Ba2] T. Bartsch, Topological Methods for Variational Problems with Symmetries, Lecture Notes in Math. 1560, Springer, Berlin Heidelberg 1993.

[Ba3] T. Bartsch, A generalization of the Weinstein-Moser theorems on periodic orbits of a Hamiltonian system near an equilibrium, preprint, Heidelberg 1994.

[Ba4] T. Bartsch, Bifurcation theorey for nonlinear eigenvalue problems, in preparation.

$[\mathrm{BaC}]$ T. Bartsch and M. Clapp, Bifurcation theory for symmetric potential operators and the equivariant cup-length, Math. Z. 204 (1990), 341-356.

[Be] V. Benci, A geometrical index for the group $S^{1}$ and some applications to the study of periodic solutions of ordinary differential equations, Commun. Pure Appl. Math. 34 (1981), 393-432.

[Bö] R. Böhme, Die Lösung der Verzweigungsgleichungen für nichtlineare Eigenwertprobleme, Math. Z. 127 (1972), 105-126.

[CaS] S. E. Capell and J. L. Shaneson, Nonlinear similarity, Ann. of Math. 113 (1981), 315-355.

[Co] C. Conley, Isolated Invariant Sets and the Morse Index, CBMS, Regional Conf. Ser. in Math. 38, AMS Providence, R.I., 1978.

[CZ] C. Conley and E. Zehnder, Morse type index theory for flows and periodic solutions for Hamiltonian systems, Comm. Pure Appl. Math. 37 (1984), 207-253.

[tD] T. tom Dieck, Transformation Groups, de Gruyter, Berlin 1987.

[D] A. Dold, Lectures on Algebraic Topology, Grundlehren der math. Wiss. 200, Springer, Berlin Heidelberg 1980.

[FR1] E. Fadell and P. H. Rabinowitz, Bifurcation for odd potential operators and an alternative topological index, J. Funct. Anal. 26 (1977), 48-67.

[FR2] E. Fadell and P. H. Rabinowitz, Generalized cohomological index theories for Lie group actions with an application to bifurcation questions for Hamiltonian systems, Inv. Math. 45 (1978), 139-174. 
[K] M. A. Krasnoselski, On special coverings of a finite-dimensional sphere, Dokl. Akad. Nauk SSSR 103 (1955), 966-969 (in Russian).

[Ma] A. Marino, La biforcazione nel caso variationale, Confer. Sem. Mat. Univ. Bari 132 (1977).

[MW] J. Mawhin and M. Willem, Critical Point Theory and Hamiltonian Systems, Springer, New York 1989.

[R1] P. H. Rabinowitz, A bifurcation theorem for potential operators, J. Funct. Anal. 25 (1977), 412-424.

[R2] P. H. Rabinowitz, Minimax Methods in Critical Point Theory with Applications to Differential Equations, CBMS, Regional Conf. Ser. in Math. 65, AMS, Providence, R.I., 1986.

[Sa] D. Salamon, Connected simple systems and the Conley index of isolated invariant sets, Trans. Amer. Math. Soc. 291 (1985), 1-41.

[Sp] E. Spanier, Algebraic Topology, McGraw-Hill, New York 1966.

[Y] C. T. Yang, On the theorems of Borsuk-Ulam, Kakutani-Yamabe-Yujòbô and Dyson, Ann. Math. 60 (1954), 262-282. 